\title{
Effect of Soy Yogurt (Lactic Acid-Fermented Okara in Soymilk) on Intestinal
}

\section{Polyamine Level in Rats}

\author{
Naohiro TAKAGI ${ }^{1}$, Kimiko TsUZUKI ${ }^{2}$ and Mitsuru FUKUdA ${ }^{3 *}$ \\ ${ }^{1}$ Graduate School of Human Environmental Sciences, Mukogawa Women's University, 6-46, Ikebiraki-cho, Nishinomiya, Hyogo 663-8558, Japan \\ ${ }^{2}$ Research Institute, Marusan-Ai Co.,Ltd., 1, Aza-Arashita, Nikki-cho, Okazaki, Aichi 444-2193, Japan \\ ${ }^{3}$ School of Human Environmental Sciences, Mukogawa Women's University, 6-46, Ikebiraki-cho, Nishinomiya, Hyogo 663-8558, Japan
}

Received July 19, 2009; Accepted May 14, 2010

Polyamines, putrescine, spermidine and spermine, are important constituents of all mammalian cells and are essentially involved in a variety of regulatory step during normal and malignant cell proliferation. Okara and soymilk fermented with Lactobacillus were combined to form soy yogurt, which is rich in polyamine content. We investigated the effect of soy yogurt on polyamine levels in intestines and blood of rats. The ingestion of soy yogurt induced the increase of mucosal polyamine level in small intestine. Although the polyamine level in cecum was increased by the ingestion of soy yogurt, the polyamine levels in large intestine and blood were not increased. It was found that the ingestion of polyamine-rich soy yogurt could affect the polyamine level in only small intestinal mucosa. The large intestine seems to regulate the polyamine level to prevent own cells from hyperproliferation. It was assumed that blood kept polyamine level constant in spite of the level changes in intestines.

Keywords: polyamine, okara, soymilk, lactic fermentation, soy yogurt, intestinal mucosa, cecum

\section{Introduction}

Okara is a byproduct generated mainly during processing of soybean. Although a great deal of okara has been treated as an industrial waste, it contains functional components including soy protein (Anderson et al., 1995, Fukui et al., 2002, Higaki et al., 2006, Iwami et al., 1986, Makino et al., 1988, Nagata et al., 1982, Takagi et al., 2008, Tanaka et al., 1989), isoflavone (Sung et al., 2004), dietary fiber (O'Toole, 1999) and polyamine (Bardocz et al., 1993). We examined the beneficial use of okara supplemented in lactic acid-fermented soymilk (soy yogurt). We already investigated the effects of soy yogurt on plasma and hepatic lipid profiles in rats (Kitawaki et al., 2007) and found that soy yogurt exhibits lipidmetabolism modification (Kitawaki et al., 2009).

In a previous paper, we reported the increase of polyamine content in okara and soymilk by lactic fermentation (Takagi et al., 2009). The polyamines, putrescine, spermidine and spermine, are important constituents of all mammalian cells and are essentially involved in a variety of regulatory steps

*To whom correspondence should be addressed.

E-mail: senjuoka@mukogawa-u.ac.jp during normal, adaptive, and malignant cell proliferation (Larquě et al., 2007, Pegg, 1988, Pegg and McCann, 2002, Tabor and Tabor, 1984). The polyamine level is correlated with biomarkers of proliferation in the large intestinal mucosa where dysregulated epithelial hyperproliferation is associated with large intestinal cancer risk (Wolter et al., 2004). Regulation of polyamine biosynthesis prevents overproduction and deficiency of polyamine in animals, and excessive amounts of polyamine are harmful, potentially leading to cancer (Pegg, 1988). The influence of dietary polyamines on human and animal are completely unknown. Wang et al. (2000) reported that dietary soy protein is associated with reduced intestinal mucosal polyamine level in rats. However, we observed the distinct response of the polyamine level between the small and large intestines by the ingestion of soy yogurt. In the present study, we investigated the effect of soy yogurt on polyamine levels in intestinal mucosa and blood.

\section{Materials and Methods}

Animals Male Sprague-Dawley (SD) rats ( $\mathrm{n}=12)$ aged 7 weeks, body weight 200-220 g (CLEA Japan, Inc. Japan), were housed individually in raised stainless steel cages with 
Table 1. Composition of experimental diet (weight \%).

\begin{tabular}{lcc}
\hline \multicolumn{1}{c}{ Ingredients } & \multicolumn{2}{c}{ Diet Group } \\
& $\mathrm{C}(\mathrm{AIN}-93 \mathrm{G})$ & $\mathrm{S}$ \\
\hline Corn starch & 39.7 & 37.2 \\
Casein & 20.0 & 11.4 \\
a-Corn starch & 13.2 & 13.0 \\
Granulated sugar & 10.0 & 10.0 \\
Soy oil & 7.0 & 2.5 \\
Cellulose powder & 5.0 & 0.8 \\
Mineral mix (AIN-93G-MX) & 3.5 & 3.5 \\
Vitamin mix (AIN-93-VX) & 1.0 & 1.0 \\
L-Cystine & 0.3 & 0.3 \\
Choline bitartarate & 0.25 & 0.25 \\
tert-Butylhydroquinone & 0.0014 & 0.0014 \\
Dried soy yogurt & 0.0 & 20 \\
Total & 100 & 100 \\
\hline
\end{tabular}

C: control, S: soy yogurt

${ }^{1}$ Japan CLEA, Osaka, Japan

${ }^{2}$ Wako Pure Chemical Industries, Osaka, Japan

${ }^{3}$ Marusan-Ai, Okazaki, Japan

Table 2. Composition of freeze-dried soy yogurt.

\begin{tabular}{lc}
\hline \multicolumn{1}{r}{ Component } & $\%$ \\
\hline Water & 5.0 \\
Protein & 37.3 \\
Fat & 23.6 \\
Carbohydrate & 8.1 \\
Dietary fiber & 21.1 \\
Mineral & 4.9 \\
\hline
\end{tabular}

wire-mesh floors and fronts under controlled conditions of light (12-h light/dark cycle), humidity (55 $\pm 7 \%$ ) and temperature $\left(23 \pm 1^{\circ} \mathrm{C}\right)$. Food and water were consumed ad libitum.

Diets The diet was based on the AIN-93G diet formula. Rats were fed AIN-93G on a pre-feeding term. The rats were divided into two groups, control group and soy yogurt group. Control group was fed AIN-93G diet. Soy yogurt group was fed AIN-93G replaced with $20 \%$ soy yogurt, as shown in Table 1 . To make soy yogurt, soymilk and powdered okara were blended in a ratio of 2 to 1 on a dry basis and fermented using Lactobacillus delburicckii subsp. Delburicckii strain of $\mathrm{SNC} 33$ at $40^{\circ} \mathrm{C}$ for $15 \mathrm{~h}$. The nutritional composition of dry soy yogurt is shown in Table 2. Diet of each group had equal nutritional composition and was administered for 4 weeks.

Intestines After the feeding period, non-fasted rats were sacrificed without affliction by diethyl ether anesthesia to collect the intestine. The entire intestine was excised immediately and placed into ice-cold $10 \mathrm{mmol} / \mathrm{l} \mathrm{PBS}, \mathrm{pH}$ 7.4. Intestinal mucosa was gently scraped with a microscope slide from each segment and stored at $-80^{\circ} \mathrm{C}$ according to the method of Wang et al. (2000) until polyamine analysis. This animal experiment was performed according to the guideline of the Animal Use Committee of Mukogawa Women's University.

Determination of polyamine Mucosal sample was suspended in PBS and homogenized. After removal of gross debris by centrifugation, 1,12-dodecanediamine was added as an internal standard, and then proteins were precipitated with a final $0.5 \mathrm{~mol} / \mathrm{L}$ perchloric acid. The protein-free supernatant (500 $\mu \mathrm{l})$ was mixed with $350 \mu \mathrm{L}$ of saturated sodium carbonate and $400 \mu \mathrm{l}$ of $37 \mathrm{mmol} / \mathrm{L}$ dansyl chloride, then the mixture were incubated at $60^{\circ} \mathrm{C}$ for $1 \mathrm{~h}$. Dansylated polyamines were extracted in toluene, dried, then redissolved in $100 \mu \mathrm{L}$ acetonitrile and finally quantified using HPLC (Wang and Higuchi, 2000). Polyamines from each sample were separated by reverse-phase HPLC with a TSK ODS-80Ts column $(4.6 \times 250$ $\mathrm{mm}$, Tosoh Corp., Japan) with $10 \mathrm{mmol} / \mathrm{L}$ heptanesulfonate buffer, $\mathrm{pH} 3.4$, using an acetonitrile gradient at a flow rate of $0.7 \mathrm{~mL} / \mathrm{min}$. Dansylated polyamines were detected using a fluorescence detector with excitation at $330 \mathrm{~nm}$ and emission at $470 \mathrm{~nm}$, and the peak areas assigned to an internal standard were used to calculate the polyamine content.

Statistical analysis Analysis involved SPSS, version $12.0 \mathrm{j}$ for Windows. Each value is shown as the mean \pm SE. All data were evaluated by one-way ANOVA and then by Student's t-test at $p<0.05$.

\section{Results and Discussion}

Food intake The food intake, initial and final body weights, and food efficiency of rats did not significantly differ between the two diet groups (Table 3). However, the difference of cecum weight depending on the content was significant between the two groups. The cecum weight/body weight of soy yogurt group was higher than that of the control group (Fig. 1).

Polyamine In our previous paper, we reported that the polyamine (putrescine, spermidine and spermine) content of fermented okara/soymilk mixture, i.e., soy yogurt (4.07 nmol polyamines/mg food), was higher compared with that of the unfermented mixture (2.68 nmol polyamines $/ \mathrm{mg}$ food) (Takagi et al., 2009); spermidine level was especially higher than those of other polyamines. Polyamines are known to play some essential roles in cell proliferation, regeneration and differentiation (Tabor and Tabor, 1984). Matsumoto et al. (2006) reported that the intestinal polyamine concentrations of elder volunteers were increased by ingestion of yogurt. In the present study, the level of polyamines (putrescine, spermidine and spermine) in small intestinal mucosa in the soy yogurt group

Table 3. Effect of test food on growth and feed efficiency ratio in rats.

\begin{tabular}{lll}
\hline & \multicolumn{1}{c}{ Control } & Soy yogurt \\
\hline Initial body weight $(\mathrm{g})$ & $297.8 \pm 4.4$ & $301.1 \pm 2.4$ \\
Final body weight $(\mathrm{g})$ & $453.9 \pm 5.5$ & $450.0 \pm 9.1$ \\
Total food intake $(\mathrm{g} / 4$ weeks) & $671.7 \pm 20.0$ & $689.9 \pm 29.8$ \\
Food efficiency & $0.23 \pm 0.005$ & $0.22 \pm 0.003$ \\
\hline
\end{tabular}

Each value is expressed as the mean $\pm \operatorname{SE}(n=6)$. 


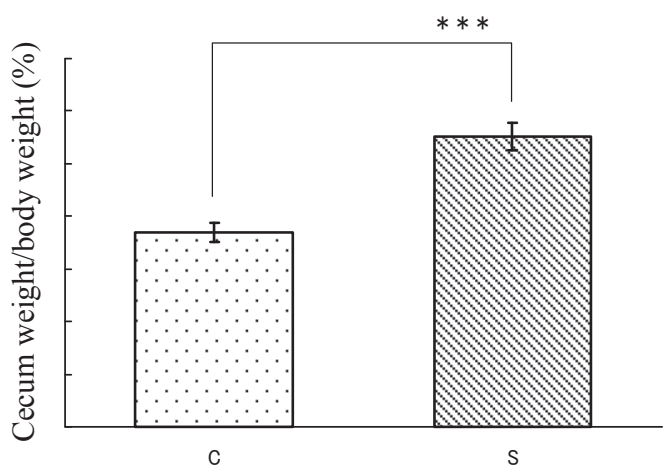

Fig. 1. Cecum weight/body weight percent of rats. Each value is expressed as the mean $\pm \mathrm{SE}(n=6)$. C: Control, S: Soy yogurt, $\quad * * * p<0.005$

was higher than that of the control group, and polyamines ingested from diets seem to be incorporated immediately after ingestion of polyamine-rich soy yogurt (Table 4). The spermidine and spermine contents in small intestine mucosa showed a significant increase in the soy yogurt group. In the small intestinal mucosa, the spermidine levels were always higher than those of putrescine and spermine. The polyamine level in large intestinal mucosa did not significantly increase (Table 4). The putrescine content in the large intestine of the soy yogurt group showed a significantly low level. Although the total polyamine level in small intestinal mucosa showed a distinct increase in the soy yogurt group, that in large intestinal mucosa of the soy yogurt group did not show any increasing tendency. On the other hand, Wang et al. (2000) reported that a soy protein diet lowered polyamine levels in small and large intestinal mucosa. This report supported our results in large intestinal mucosal polyamines, whereas the polyamine level in small intestinal mucosa was different from our results. Increasing the level of polyamines in the diet by soy yogurt intake would lead to incorporation of polyamines in small intestinal mucosa, despite inhibition of polyamine absorption with soy protein. Excess polyamine in large intestinal cells accelerates tumorigenesis (Wolter et al., 2004). The large intestine seems to regulate the polyamine level to prevent its own cells from hyperproliferation.

The cecum polyamine content in the soy yogurt group was higher than that in the control group. Cecum microorganisms in rats that ingested soy yogurt might be increased and polyamine was produced (Table 4), as cecum weight/body weight of the soy yogurt group was significantly higher than that of control group (Fig. 1). As reported in our previous paper (Takagai et al., 2009), the ingestion of guar gum or soy yogurt increased cecum weight and the level of polyamines. Bifidobacteria in cecum was increased to more than 100 fold by soy yogurt ingestion, whereas the remarkable increase of Bacteroides involved in polyamine synthesis was not detected (unpublished data). However, Noack et al. (1998) observed a vigorous increase of both Bfidobacteria and Bacteroides in rats that had ingested guar gum. As Bifidobacteria scarcely participates in polyamine biosynthesis in rats (Noak et al., 1998), we have not yet specified the bacteria producing polyamines in the cecum. However, in the present study, changes in cecum polyamine content in rats ingested soy yogurt could be affected by various microorganisms.

In the present study, there was no significant difference between the blood polyamine levels of the two groups (Table 4). Brodal et al. (1999) also reported that polyamine intake did not exhibit any effect on blood polyamine levels. Moreover, their results suggested that blood polyamine levels were regulated by intestinal absorption and catabolism. Delzenne et al. (2000) reported that the high level of polyamine in rat cecum did not affect the level in the portal blood.

These results exhibited that the ingestion of soy yogurt induced the increase of the mucosal polyamine level in small intestine whereas the levels in large intestinal mucosa and blood did not increase. The ingestion of soy yogurt induced the absorption of polyamines in small intestinal mucosa. Thus the increase in mucosal polyamine level should enhance intestinal mucosal barrier function as reported by Guo et al. (2003). On the other hand, the large intestine seems to regulate the polyamine level to prevent its own cells from hyperproliferation and tumorigenesis. It was assumed that the blood kept the polyamine level constant despite the level changes in intestinal mucosa. Thus, a large amount of polyamine intake may be required to increase the polyamine level in blood.

Table 4. Polyamine levels in intestine, blood and cecum of rats.

\begin{tabular}{lcc}
\hline \multicolumn{1}{c}{ Polyamines } & Control & Soy yogurt \\
\hline Small intestine (nmol/mg protein) & & \\
$\quad$ Putrescine & $0.8 \pm 0.2$ & $1.3 \pm 0.2$ \\
Spermidine & $9.9 \pm 2.0$ & $15.2 \pm 1.3^{*}$ \\
$\quad$ Spermine & $4.7 \pm 0.6$ & $6.5 \pm 0.3^{*}$ \\
$\quad$ Total & $15.4 \pm 3.2$ & $23.1 \pm 1.8^{*}$ \\
Large intestine (nmol/mg protein) & & \\
$\quad$ Putrescine & $1.1 \pm 0.2$ & $0.6 \pm 0.0^{*}$ \\
Spermidine & $8.6 \pm 0.6$ & $9.2 \pm 0.5$ \\
Spermine & $5.7 \pm 0.6$ & $5.4 \pm 0.3$ \\
Total & $15.4 \pm 1.1$ & $15.1 \pm 0.7$ \\
Blood (nmol/1) & & \\
$\quad$ Putrescine & $2.3 \pm 0.1$ & $2.4 \pm 0.1$ \\
Spermidine & $2.8 \pm 0.3$ & $2.6 \pm 0.1$ \\
Spermine & $0.3 \pm 0.1$ & $0.3 \pm 0.1$ \\
Total & $5.3 \pm 0.3$ & $5.3 \pm 0.2$ \\
Cecum (nmol/mg cecum content) & & \\
$\quad$ Putrescine & $0.4 \pm 0.1$ & $0.1 \pm 0.0^{*}$ \\
Spermidine & $2.1 \pm 0.2$ & $3.7 \pm 0.2^{* * *}$ \\
Spermine & $0.3 \pm 0.0$ & $0.3 \pm 0.0$ \\
Total & $2.7 \pm 0.3$ & $4.1 \pm 0.2^{*}$ \\
\hline
\end{tabular}

Each value is expressed as the mean $\pm \mathrm{SE}(n=6)$. Asterisks show significantly different values from that of the control by Student's $t$-test. $(* p<0.05, * * * p<0.005)$ 


\section{References}

Andereson, J.W., Johnstone, B.M. and Cook-Newell, M.E. (1995). Meta-analysis of the effects of soy protein intake on serum lipids. N. Engl. J. Med., 333, 276-282.

Bardocz, S., Grant, G., Brown, D. S., Ralph, A. and Pusztai, A. (1993). Polyamine in food-implications for growth and health. $J$. Nutr. Biochem., 4, 66-71.

Brodal, B.P., Eliassen, K.A., Rönning, H. and Osmundsen, H. (1999). Effect of dietary polyamines and clofibrate on metabolism of polyamines in the rat. J. Nutr. Biochem., 10, 700-708.

Delzenne, N.M., Kok, N., Deloyer, P. and Dandrifosse, G. (2000). Dietary fructans modulate polyamine concentration in the cecum of rats. J. Nutr., 130, 2456-2460.

Fukui, K., Tachibana, N., Wanezaki, S., Tsuzaki, S., Takamatsu, K., Yamamoto, T., Hashimoto, Y. and Shimoda, T. (2002). Isoflavonefree soy protein prepared by column chromatography reduces plasma cholesterol in rats. J. Agric. Food Chem., 50, 5717-5721.

Guo, X., Rao, J.N., Liu, L., Zou, T.T., Turner, D.J., Bass, B.L. and Wang, J.Y. (2003). Regulation of adherens junctions and epithelial paracellular permeability: a novel function for polyamines. Am. J. Physiol., 285, C1174-C1187.

Higaki, N., Sato, K., Suda, H., Suzuka, T., Komori, T., Saeki, T., Nakamura, Y., Ohtsuki, K., Iwami, K. and Kanamoto, R. (2006). Evidence for the existence of a soybean resistant protein that captures bile acid and stimulates its fecal excretion. Biosci. Biotech. Biochem., 70, 2844-2852.

Iwami, K., Sakakibara, K. and Ibuki, F. (1986). Involvement of postdigestion 'Hydrophobic' peptides in plasma cholesterol-lowering effect of dietary plant proteins. Agric. Biol. Chem., 50, 12171222.

Kitawaki, R., Takagi, N., Iwasaki, M., Asao, H., Okada, S. and Fukuda, M. (2007) Plasma cholesterol-lowering effects of soymilk and okara treated by lactic acid fermentation in rats. Nippon Shokuhin Kagaku Kogaku Kaishi, 54, 379-382 (in Japanese).

Kitawaki, R., Nishimura, Y., Takagi, N., Iwasaki, M., Tsuzuki, K. and Fukuda, M. (2009). Effect of lactobacillus fermented soymilk and soy yogurt on hepatic lipid accumulation in rats fed cholesterol-free diet. Biosci. Biotech. Biochem., 73, 1484-1488.

Larquĕ, E., Sabater-Molina, M. and Zamora, S, (2007). Biological significance of dietary polyamines, Nutrition, 23, 87-95.

Makino, A., Nakashima, H., Minami, K., Moriyama, R. and Takao, S. (1988). Bile acid-binding protein from soybean seed: isolation, partial characterization and insulin- stimulating activity. Agric. Biol. Chem., 52, 803-809.

Matsumoto, M. and Benno, Y. (2006). Anti-inflammatory metabolite production in the gut from the consumption of Probiotic yogurt containing Bifidobacterium animalis subsp. lactis LKM512. Biosci. Biotechnol. Biochem., 70, 1287-1292.

Milovic, V. (2001). Polyamines in the gut lumen: bioavailability and biodistribution. Eur. J. Gastroenterol. Hepatol., 13, 1021-1025.

Nagata, Y., Ishiwaki, N. and Sugano, M. (1982). Studies on the mechanism of antihypercholesterolemic action of soy protein and soy protein-type amino acid mixtures in relation to the casein counterparts in rats. J. Nutr., 112, 1614-1625.

Noak, J., Kleesen, B., Proll, J., Dongowski, G. and Biaut M. (1998). Dietary guar gum and pectin stimulate intestinal microbial polyamine synthesis in rats. J. Nutr., 128, 1385-1391.

O'Toole, D.K. (1999). Characteristics and use of okara, the soybean residue from soy milk production - A review. J. Agric. Food. Chem., 47, 363-371.

Pegg, A.E. (1988). Polyamine metabolism and its importance in neoplastic growth and as a target for chemotherapy. Cancer. Res., 48, 759-774.

Pegg, A.E. and McCann, P.P. (2002). Polyamine metabolism and function. Am. J. Physiol., 243. C212-C221.

Sung, J.H., Lee, S.J., Park, K.H. and Moon, W. (2004). Isoflavones inhibit 3-hydroxy-3-methylglutaryl coenzyme A reductase in vitro. Biosci. Biotech. Biochem., 68, 428-432.

Tabor, C.W. and Tabor, H. (1984). Polyamines. Ann. Rev. Biochem., 53, 749-790.

Takagi, N., Kitawaki, R., Nishimura, Y., Iwasaki, M., Tsuzuki, K., Horiuchi, R. and Fukuda, M. (2008). Effect of okara/soymilk fermented by lactic acid bacteria on fecal bile acid excretion of rats. Nippon Shokuhin Kagaku Kougakkaishi, 55, 597-601 (in Japanese).

Takagi, N., Kitawaki, R., Nishimura, Y., Harada, T., Iwasaki, M., Tsuzuki, K. and Fukuda, M. (2009). Effect of okara and soymilk mixture fermented with lactic acid bacteria on short chain fatty acid and polyamine concentration in rat cecum. Nippon Shokuhin Kagaku Kougaku Kaishi, 56, 585-590 (in Japanese).

Tanaka, K. and Sugano, M. (1989). Effects of modification of the arginine/lysine ratio of dietary proteins on absorption and turnover of cholesterol in rats. Agric. Biol. Chem., 53, 1351-1356.

Wang, W and Higuchi, C.M. (2000). Dietary soy protein is associated with reduced intestinal mucosal polyamine concentration in male Wister rats. J. Nutr., 130, 1815-1820.

Wolter, F., Ulrich, S. and Stein, J. (2004). Molecular mechanisms of the chemopreventive effects of resveratrol and its analogs in colorectal cancer: key role of polyamines? J. Nutr., 134, 3219 3222 . 